\title{
Case Report Form (CRF) Design Made Easy: An Evaluation of Clinical Data Acquisition Standards Harmonization (CDASH) in Use
}

\author{
Motohiko Adomi ${ }^{1}$, Satoshi Ueno ${ }^{2}$, Masahiko Gosho ${ }^{3}$, Yukiko Wagatsuma ${ }^{3}$ and Masafumi Okada ${ }^{4}$ \\ ${ }^{1}$ School of Medicine, University of Tsukuba, Japan \\ ${ }^{2}$ Faculty of Medicine, University of Tsukuba, Japan \\ ${ }^{3}$ Department of Clinical Trial and Clinical Epidemiology, Faculty of Medicine, University of Tsukuba, Japan \\ ${ }^{4}$ University hospital Medical Information Network (UMIN), Japan
}

\begin{abstract}
Abbreviations: CDISC: Clinical Data Interchange Standards Consortium; CDASH: Clinical Data Acquisition Standards Harmonization; CRF: Case Report Form; CDM: Clinical Data Manager; PI: Principal Investigator; SDTM: Study Data Tabulation Model; UICC: Union For International Cancer Control; JSCCR: Japanese Society For Cancer Of The Colon And Rectum
\end{abstract}

\section{Introduction}

The standardized formats of clinical research data have been developed and established by the Clinical Data Interchange Standards Consortium (CDISC), which is addressing standardization in order to support the acquisition, exchange, submission, and archive of clinical data from individual trials and their metadata [1]. CDISC has developed the Clinical Data Acquisition Standards Harmonization (CDASH), which is a standard for data collection [2-4]. Data reliability, integrity, and exchangeability for clinical research depend greatly on having accurate and uniform definitions of the data items to be collected and the design of case report forms (CRF). The use of CDASH is very helpful for standardizing the items and data formats in a CRF for any trial across different research institutions.

Although CDASH is considered as a breakthrough to control the quality of CRFs, its implementation has scarcely been reported for actual clinical trials and its practicality has not been sufficiently discussed. Therefore, we present a report of using CDASH to design a $\mathrm{CRF}$, an evaluation of its usefulness, and a discussion of our experience and findings.

\section{Methodology}

In this study, a medical student, with no experience of clinical trials, was given the task of designing a CRF using CDASH in order to evaluate its usefulness. The process of the study, which was implemented by only one student, is composed of 3 stages; training, production, and evaluation.

First, in the training stage, the student went through a training process to learn about clinical trials, $\mathrm{CRF}$ and $\mathrm{CDASH}$. An experienced clinical data manager (CDM) lectured the student about clinical trials, how CRF and CDASH work in clinical trials, and how to use each standard. The training course was held for 2-3 hours, every 2 weeks, for 2 months. After finishing the course, the student read 3 manuals for self-study: CDASH Standard [2], CDASH User Guide [3] and Library of Example CRFs [4].

Next, in the production stage, the student was given the protocol and independently designed the CRF. The applied protocol was of a colorectal cancer clinical trial conducted in the University of Tsukuba Hospital and the Tsukuba Medical Center Hospital. This study was an open-label, single arm, therapeutic exploratory, phase I study. In total, 100 patients with colorectal cancer will be recruited from the University of Tsukuba Hospital and the Tsukuba Medical Center Hospital, and enrolled into the study if they meet the eligibility criteria. Ethical approvals were obtained from the Research ethics committees at the two centers. Patients enrolled in this study will receive daily clinical practice, be collected clinical data (demographics, medical history, laboratory examinations, previous/concomitant medications and imaging test). This study aims to evaluate the immunosuppressive function in colorectal cancer patients. No particular criteria were used when choosing the protocol, because CDASH describes the basic data collection fields for 18 general domains such as 'Demographics' and 'Adverse Events', which are common to almost all protocols. A Principal Investigator (PI) in the trial and the CDM decided the data items of CRF. After the student received the data items from the CDM, the student searched CDASH for the appropriate parts to explain each data item and mapped the variables to the CRF. The data items defined in the protocol are listed in Table 1. When the items were not defined in CDASH, Study Data Tabulation Model (SDTM) standards were used instead [5]. For more detailed process of CRF design, see Appendix 1.

Finally, in the evaluation stage, the CDM evaluated the CRF designed by the student. The variables mapped in the CRF were compared with those defined in CDASH. To evaluate the performance of the designed CRF, we analyzed its concordance with CDASH. When the CDISC-defined part of the variable was identical, the item was counted as concordant. For detailed concept of this definition, see Appendix 2.

\section{Results}

The data items defined in the protocol, the domain of the data items, and the named variables by the student are listed in Table 1. A total of 52 data items were defined in the protocol. All the items were correctly categorized into 9 domains (Common Identifier Variables, Disposition, Demographics, Inclusion/Exclusion Criteria Not Met, Laboratory Test Results, Prior/Concomitant Medications, Substance Use, Tumor Identification and Tumor Results) and were accurately reflected in the CRF. Of the items, 30 were found in the CDASH manual and the remaining 22 were not; for these items categorized in domains 'Tumor Identification' and 'Tumor Results', we referred to SDTM. All the 52 items were named by the student according to CDASH or SDTM. However, of 52 items, 6 items categorized in the 'Inclusion/Exclusion Criteria Not Met' domain were incorrectly named. Excluding this domain, the concordance proportions of the other 8 domains were 100\% (Table 1). The concordance proportions by each domain were as follows: Common Identifier Variables: 100\%

*Corresponding author: Satoshi Ueno, Faculty of Medicine, University of Tsukuba 1-1-1 Tennodai, Tsukuba Ibaraki 305-8575, Japan, Tel: +81-29-853-3415; Fax: +81-29853-3258; E-mail: satoshi.ueno@md.tsukuba.ac.jp

Received October 16, 2015; Accepted November 17, 2015; Published November 19,2015

Citation: Ueno S (2015) Case Report Form (CRF) Design Made Easy: An Evaluation of Clinical Data Acquisition Standards Harmonization (CDASH) in Use. Pharmaceut Reg Affairs 4: 153. doi:10.4172/2167-7689.1000153

Copyright: (c) 2015 Ueno S. This is an open-access article distributed under the terms of the Creative Commons Attribution License, which permits unrestricted use, distribution, and reproduction in any medium, provided the original author and source are credited. 
Citation: Ueno S (2015) Case Report Form (CRF) Design Made Easy: An Evaluation of Clinical Data Acquisition Standards Harmonization (CDASH) in Use. Pharmaceut Reg Affairs 4: 153. doi:10.4172/2167-7689.1000153

Page 2 of 4

\begin{tabular}{|c|c|c|c|c|}
\hline Contents of CRF & Domain of CDASH & Variable name of CDASH & Variable name (student) & $\begin{array}{c}\text { Concordance } \\
\text { proportion }\end{array}$ \\
\hline What is the site identifier? & $\begin{array}{c}\text { Common Identifier } \\
\text { Variables }\end{array}$ & SITEID & SITEID & $100 \%(3 / 3)$ \\
\hline What is the subject identifier? & & SUBJID & SUBJID & \\
\hline What is the investigator identifier? & & INVID & INVID & \\
\hline
\end{tabular}

What was the date of informed consent?

Disposition

DSSTDAT

DSSTDAT

Demographics

AGE

SEX

What is the sex of the subject?

Inclusion Criteria 1

Inclusion Criteria 2

Inclusion Criteria 3

Inclusion Criteria 4

Inclusion Criteria 5

Exclusion Criteria 1

What was the lab specimen collection schedule date?

What was the lab specimen collection date?

What was the result of the test?

What were the units for the result?

Did the subject take chemotherapy?

What was the term for the listed oral chemotherapy taken?

What was the term for any other oral chemotherapy taken?

What was the term for the listed intravenous chemotherapy taken?

What was the term for any other listed intravenous chemotherapy taken?

Did the subject take steroids?

Has the subject ever used tobacco?

What was the duration of tobacco use?

What was the frequency of tobacco use?

Has the subject ever used alcohol?

What was the duration of alcohol use?

What was the amount of alcohol used?

What was the frequency of alcohol use?
Laboratory Test Results

LBDAT

LBDAT

Inclusion/Exclusion Criteria IEORRES Not Met

IEORRES

IEORRES

IEORRES

IEORRES

IEORRES

LBORRES

LBORRESU

Prior/Concomitant Medications

CMOCCUR

CMTRT

CMTRT

CMTRT

CMTRT

CMOCCUR

Substance Use

SUNCF

SUCDUR

SUDOSFRQ

SUNCF

SUCDUR

SUDSTXT

SUDOSFRQ
DSSTDAT_informedconsent

$100 \%(2 / 2)$

DSSTDAT_registration

AGE

$100 \%(2 / 2)$

SEX

INC_001

$0 \%(0 / 6)$

INC_002

INC_003

INC_004

INC_005

EXC_001

LBDAT_specimen

$100 \%(4 / 4)$

LBDAT_tumor_marker

LBORRES_tumor_marker

LBORRESU tumor_marker

CMOCCUR_chemotherapy

$100 \%(6 / 6)$

CMTRT_chemotherapy_ORAL

CMTRT_chemotherapy_ORAL_others

CMTRT_chemotherapy_INTRAVENOUS

CMTRT_chemotherapy_INTRAVENOUS_ others

CMOCCUR_steroid

SUNCF_TOBACCO

$100 \%(7 / 7)$

SUDOSFRQ_TOBACCO

SUNCF_ALCOHOL

SUCDUR ALCOHOL

SUDSTXT_ALCOHOL

SUDOSFRQ_ALCOHOL 
Citation: Ueno S (2015) Case Report Form (CRF) Design Made Easy: An Evaluation of Clinical Data Acquisition Standards Harmonization (CDASH) in Use. Pharmaceut Reg Affairs 4: 153. doi:10.4172/2167-7689.1000153

Page 3 of 4

Where was the location of the tumor?

What was the longest diameter of the tumor?

What was the width of the tumor? (Perpendicular to the longest diameter)

What was the clinical assessment of $T^{\star *}$ of the tumor according to the Japanese Classification of Colorectal Carcinoma? (JSCCR)

What was the clinical assessment of $\mathrm{N}^{\star *}$ of the tumor according to JSCCR?

What was the clinical assessment of $M^{\star *}$ of the tumor according to JSCCR?

What was the stage of the tumor? (Clinically assessed according to JSCCR)

What was the pathological assessment of $T$ of the tumor according to JSCCR?

What was the pathological assessment of $\mathrm{N}$ of the tumor according to JSCCR?

What was the pathological assessment of $M$ of the tumor according to JSCCR?

What was the stage of the tumor? (Pathologically assessed according to JSCCR?)

What was the histopathological type of the tumor according to JSCCR?

What was the clinical assessment of $T^{\star *}$ of the tumor according to the International Union Against Cancer (UICC) TNM Classification of Malignant Tumours?

What was the clinical assessment of $\mathrm{N}^{* *}$ of the tumor according to UICC?

What was the clinical assessment of $\mathrm{M}^{* *}$ of the tumor according to UICC?

What was the stage of the tumor? (Clinically assessed according to UICC)

What was the pathological assessment of $T$ of the tumor according to UICC?

What was the pathological assessment of $\mathrm{N}$ of the tumor according to UICC?

What was the pathological assessment of $M$ of the tumor according to UICC?

What was the stage of the tumor? (pathologically assessed according to UICC)

What was the histopathological grade of the tumor according to UICC?

What was the macroscopic classification?
Tumor Identification * TULOC

TULOC

$100 \%(1 / 1)$

Tumor Results **** TRORRES

TRORRES

TRORRES

TRORRES

TRORRES

TRORRES

TRORRES

TRORRES

TRORRES

TRORRES

TRORRES

TRORRES

TRORRES

TRORRES

TRORRES

TRORRES

TRORRES

TRORRES

TRORRES

TRORRES

TRORRES $\begin{array}{cc}\text { TRORRES_longest_diameter_along__ } & 100 \% \\ \text { major_axis } & (21 / 21)\end{array}$

TRORRES_longest_diameter_ perpendicular_to_previous_one TRORRES_clinical_T

TRORRES_clinical_N

TRORRES_clinical_M

TRORRES_clinical_stage

TRORRES_pathological_T

TRORRES_pathological_N

TRORRES_pathological_M

TRORRES_pathological_stage

TRORRES_pathological_histological_stage

TRORRES_UICC_clinical_T

TRORRES_UICC_clinical_N

TRORRES_UICC_clinical_M

TRORRES_UICC_clinical_stage

TRORRES_UICC_pathological_T

TRORRES_UICC_pathological_N

TRORRES_UICC_pathological_M

TRORRES_UICC_pathological_stage

TRORRES_UICC_pathological_ differenciation

TRORRES_gross_stage

* 'Tumor Identification'and 'Tumor Results' domains are defined in SDTM [5].

** T, N, and M are defined as 'The extent of the primary tumour,' 'The absence or presence and extent of regional lymph node metastasis' and 'The absence or presence of distant metastasis' according to International Union Against Cancer (UICC) TNM Classification of Malignant Tumours[6].

*** The contents of CRF in the 'Tumor Results' domain are defined according to International Union Against Cancer (UICC) TNM Classification of Malignant Tumours or Japanese Classification of Colorectal Carcinoma by Japanese Society for Cancer of the Colon and Rectum (JSCCR) [6,7].

Table 1: The data items defined in the protocol.

(3/3); Disposition: 100\% (2/2); Demographics: 100\% (2/2); Inclusion/ Exclusion Criteria Not Met: 0\% (0/6); Laboratory Test Results: $100 \%$ (4/4); Prior/Concomitant Medications: 100\% (6/6); Substance Use: 100\% (7/7); Tumor Identification: 100\% (1/1); and Tumor Results: $100 \%(21 / 21)$.

\section{Discussion}

Surprisingly, the concordance proportion was very high, except for the 'Inclusion/Exclusion Criteria Not Met' domain. With regard to this domain, the mismatch may have occurred due to the student's misunderstanding of Inclusion/Exclusion Criteria in CDASH and the variable name that the student used for this domain was found in the Library of Example CRFs published by CDISC. 'Inclusion/
Exclusion Criteria Not Met' domain is different from other domains due to insufficient explanation about mapping the variables. Because $\mathrm{CDASH}$ does not define specific variables regarding this domain, it is difficult for beginners to correctly map them. On the other hand, the explanations to map unambiguous variables of the other 8 domains are very easy to understand, even for beginners. The description of the variables in 'Inclusion/Exclusion Criteria Not Met' domain should be modified to be user-friendly.

The incompleteness of the CDASH standards themselves could decrease the concordance. In our case, some data items in the protocol corresponded to the Union for International Cancer Control (UICC) guidelines and Japanese Classification of Colorectal Carcinoma (JSCCR) [6,7]. These data items were categorized in 'Tumor Results' 
domain. However, detailed data items such as What was the stage of the tumor? (Clinically assessed according to UICC) were not provided in CDASH and SDTM standards. In other words, disease specific variables are not provided in these standards. Therapeutic Area Standards are currently being developed to provide variables and items in each disease area [8]. While some standards are already available, the standardization for colorectal cancer has not yet been completed.

\section{Limitations}

There are two limitations in this study. First, the limitation of this study is it did not meet Tier 2 of the 2 levels of conformance to CDASH. Tier 1 evaluates the conformance at the CRF level and Tier 2 evaluates at the operational level $[2,3]$. We met only the Tier 1 level of conformance, because we did not apply the designed CRF to actual data collection. Second, because this is a case report, it is necessary to increase the number of subjects in experiment to obtain generalizable result.

\section{Conclusion}

Through our study we have confirmed that CDASH standard is useful to design CRFs, especially to design general domains such as 'Demographics' and 'Adverse Event'. It is also easy for researchers who do not have much experience in clinical trials to use the CDASH standard.

\section{Funding}

This work was supported by Pfizer Health Research Foundation [Grant Number 13-9-049 to SU] and JSPS KAKENHI [Grant Number $15 \mathrm{~K} 15218$ to $\mathrm{MO}]$.

\section{Declaration of Conflicting Interests}

The Authors declare that there is no conflict of interest.

\section{Acknowledgments}

We would like to thank Thomas Mayers (Medical English Communications Center, University of Tsukuba) for grammatical review and advice.

\section{References}

1. (2015) CDISC Vision and Mission, CDISC

2. (2011) CDASH v1.1 Standard, CDISC.

3. (2012) CDASH User Guide v1, CDISC.

4. (2015) Library of CDASH CRF Examples, CDISC.

5. (2013) Study Data Tabulation Model Implementation Guide v3.2, CDISC.

6. Leslie S, Mary G, Christian W (2009) TNM Classification of Malignant Tumours (7thedn) Wiley-Blackwell, New York.

7. (2013) Japanese Classification of Colorectal Carcinoma. (8thedn) Japanese Society for Cancer of the Colon and Rectum, Kanehara \& Co., Ltd., Tokyo.

8. (2015) Therapeutic Area Standards, CDISC. 